\title{
The novel photon detectors based on MPGD technologies for the upgrade of COMPASS RICH-1
}

\author{
J.Agarwala $^{a}$, M.Alexeev ${ }^{b}$, C.D.R.Azevedo ${ }^{c}$, R.Birsa $^{d}{ }$, F.Bradamante ${ }^{e}$, A.Bressan ${ }^{e}$, \\ C.Chatterjee $^{e}$, M.Chiosso $^{b}{ }$, A.Cicuttin ${ }^{a}$, P.Ciliberti ${ }^{e}$, M.L.Crespo ${ }^{a}$, S.Dalla Torre ${ }^{d}$, \\ S.S.Dasgupta ${ }^{d}$, O.Denisov ${ }^{f}$, M.Finger $^{g}$, M.Finger Jr. $^{g}$, B.Gobbo ${ }^{d}$, M.Gregori ${ }^{d}$,

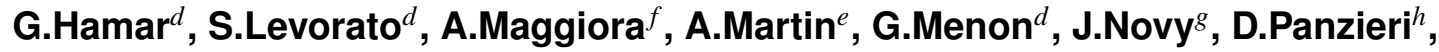 \\ F.A.B.Pereira ${ }^{c}$, C.A.Santos ${ }^{d}$, G.Sbrizzai ${ }^{i}$, M.Slunecka ${ }^{g}$, K.Steiger $^{i}$, L.Steiger ${ }^{i}$, \\ M.Sulc $^{i}$, F.Tessarotto ${ }^{*} \dagger$, J.F.C.A.Veloso ${ }^{c}$, Y.Zhao $^{d}$ \\ ${ }^{a}$ INFN, Sezione di Trieste and Abdus Salam ICTP, Trieste, Italy \\ ${ }^{b}$ INFN, Sezione di Torino and University of Torino, Torino, Italy \\ ${ }^{c} I 3 N$ - Physics Department, University of Aveiro, Aveiro, Portugal \\ ${ }^{d}$ INFN, Sezione di Trieste, Trieste, Italy \\ ${ }^{e}$ INFN, Sezione di Trieste and University of Trieste, Trieste, Italy \\ ${ }^{f}$ INFN, Sezione di Torino, Torino, Italy \\ ${ }^{g}$ Charles University, Prague, Czech Republic and JINR, Dubna, Russia \\ ${ }^{h} I N F N$, Sezione di Torino and University of East Piemonte, Alessandria, Italy \\ ${ }^{i}$ Technical University of Liberec, Liberec, Czech Republic \\ E-mail: fulvio.tessarotto@ts.infn.it
}

The RICH-1 Detector of the COMPASS experiment at CERN SPS has undergone an important upgrade in 2016. Four new photon detectors, based on MPGD technology and covering a total active area larger than $1.2 \mathrm{~m}^{2}$ have replaced the previously used MWPC-based photon detectors. The new detector architecture, resulting from a dedicated, eight years long, $R \& D$ program, consists in a hybrid MPGD combination of two THGEMs and a Micromegas stage; the first THGEM, coated with a CsI layer, acts as a reflective photocathode. The signals are extracted from the anode pads by capacitive coupling and read-out by analog front-end electronics based on the APV25 chip. The new COMPASS RICH-1 photon detectors are described in detail: the detector design, the engineering aspects, the mass production, and the quality assessment are discussed. The assembly of the MPGD components and the installation of the new detectors are illustrated together with the main aspects of the commissioning. Preliminary indication of performance results are also presented.

5th International Conference on Micro-Pattern Gas Detectors (MPGD2017)

22-26 May, 2017

Philadelphia, USA

\footnotetext{
*Speaker.

${ }^{\dagger}$ corresponding author
} 


\section{Introduction}

The COMPASS RICH-1 [1] detector is a large gaseous Ring Imaging Cherenkov Counter providing hadron identification in the range of momenta between 3 and $60 \mathrm{GeV} / \mathrm{c}$, over a large angular acceptance ( $\pm 200 \mathrm{mrad})$, for the COMPASS Experiment [2] at CERN SPS.

It consists of a $3 \mathrm{~m}$ long $C_{4} F_{10}$ radiator, a $21 \mathrm{~m}^{2}$ large focusing VUV mirror surface and Photon Detectors (PDs) covering a total active area of $5.5 \mathrm{~m}^{2}$. Three photodetection technologies are used in RICH-1: Multi Wire Proportional Chambers (MWPCs) with CsI photocathodes, Multi Anode Photo-Multipliers Tubes (MAPMTs) and Micro Pattern Gaseous Detectors (MPGDs) based PDs.

COMPASS RICH-1 was designed and built between 1996 and 2001 and is in operation since 2002. The whole photodetection surface was originally equipped with MWPCs hosting 16 CsIcoated photocathodes of about $600 \times 600 \mathrm{~mm}^{2}$ active area.

Inspite of their good performance, MWPCs with CsI-coated photocathodes have limitations in terms of maximum effective gain, time response, rate capability and aging of the CsI photoconverter. In 2006, to cope with the high particle rates of the central region, 4 of the 16 CsI-coated photocathodes were replaced by detectors consisting of arrays of MAPMTs coupled to individual fused silica lens telescopes.

In parallel, an extensive R\&D program [3], aimed to develop MPGD-based large area PDs, established a novel hybrid technology combining MicroMegas (MM) and THick Gas Electron Multipliers (THGEMs)

In 2016 COMPASS RICH-1 was upgraded by replacing other 4 MWPCs-based PDs with novel detectors, resulting from the newly developed MPGD hybrid technology [4]. The description of all aspects of this upgrade is the purpose of the present article.

\section{The development of THGEM-based PDs.}

MPGD-based PDs had previously been developed and successfully used by PHENIX-HBD [5], in the form of triple GEMs operating in windowless mode, in pure $\mathrm{CF}_{4}$. The wide wavelength range (108-200 nm) Cherenkov photons are converted by the CsI layer on the first GEM and large (6.2 $\mathrm{cm}^{2}$ ) readout pads provide collective signals from several (5 to 10) photons. To achieve an efficient detection of single photons in a narrower wavelength range a different approach, based on the THGEM technology was chosen by the dedicated R\&D program for the COMPASS RICH upgrade and also planned by the ALICE VMHPID project [6].

THGEMs [7] are gaseous electron multipliers derived from the GEM design, scaling the geometrical parameters and using standard Printed Circuit Boards (PCBs), where holes are obtained by drilling. THGEMs with different geometrical parameters have been extensively characterized and their performance as electron multipliers and as reflective photocathodes has been studied in detail [8]. THGEM-based PDs have been built and operated in Ar-based and in Ne-based gas mixtures: gain values in the range of $10^{5}-10^{6}$ and stable operation have been obtained for small-size prototypes of various configurations [9]. Time resolution values below $10 \mathrm{~ns}$ [10] are typical. Obtaining the same performance in terms of gain and stability with large or medium size $(300 \times 300$ $\mathrm{cm}^{2}$ ) triple THGEM PDs was challenging: a study of the origin of non-uniformity of the detector response and the spark rates as well as the performance of different PD configurations provided 
a specific procedure for large area THGEM production [11], quality assessment and configuration optimization. The Ion Back-Flow (IBF), which in a standard triple THGEM configuration approaches $30 \%$, can be reduced by a complete misalignment of the holes in different layers and using unbalanced values of the electric field in the transfer regions between THGEMs [12] and, more efficiently, by using a MM as last gas amplification stage: this hybrid MM and THGEM detector architecture was tested and provided better results in terms of PD performance and stability for large area prototypes [13] and was thus chosen for the COMPASS RICH-1 upgrade.

\section{The COMPASS hybrid PD architecture and components}

Each of the four new COMPASS PDs covers an active area of $\sim 600 \times 600 \mathrm{~mm}^{2}$ and is formed by two identical modules $\left(600 \times 300 \mathrm{~mm}^{2}\right)$, arranged side by side.

The hybrid module (Fig. 1) consists of two planes of wires, two layers of THGEMs and a Micromegas on a pad-segmented anode. UV light sensitivity is provided by a $300 \mathrm{~nm}$ thick CsI layer on the top of the first THGEM electrode, which acts as a reflective photocathode for VUV photons. A $600 \times 600 \mathrm{~mm}^{2}, 5 \mathrm{~mm}$ thick fused silica window separates the PD gas volume from the RICH radiator volume.

The wire planes, called Protection and Drift, are made of $100 \mu \mathrm{m}$ diameter $\mathrm{Cu}-\mathrm{Be}, \mathrm{Au}$-coated wires, $\sim 600 \mathrm{~mm}$ long, with a pitch of $4 \mathrm{~mm}$. The Protection wire plane is located at $4.5 \mathrm{~mm}$ from the fused silica window, it is set at ground potential and collects the electrons from the volume above the THGEMs; the Drift wire plane is placed at $4 \mathrm{~mm}$ from the CsI coated THGEM (and 38.5 $\mathrm{mm}$ from the Protection plane) and is biased to the voltage which maximizes the extraction and collection of the photoelectrons while repelling the ionization electrons.

All THGEMs (Fig. 3, left) have the same geometrical parameters: they are $470 \mu \mathrm{m}$ thick (400 $\mu \mathrm{m}$ dielectric and $2 \times 35 \mu \mathrm{m} \mathrm{Cu}$ ), $581 \mathrm{~mm}$ long and $287 \mathrm{~mm}$ wide; their holes have 400 $\mu \mathrm{m}$ diameter, $800 \mu \mathrm{m}$ pitch and no rim. Holes located along the external borders have $500 \mu \mathrm{m}$ diameter. The top and bottom electrodes of each THGEM are segmented in 12 parallel sectors (24 $\mathrm{mm}$ wide, apart from the border ones, which are $17 \mathrm{~mm}$ wide), separated by $0.7 \mathrm{~mm}$ clearance area. Each sector of the THGEMs is electrically decoupled from the others by a $1 G \Omega$ resistor; six consecutive sectors, grouped together, are fed by a specific high voltage power supply channel. The two THGEM layers are mounted at a distance of $3 \mathrm{~mm}$, in a configuration of complete hole misalignment, to achieve the maximum charge spread; a $5 \mathrm{~mm}$ gap separates the second THGEM from the MM (Fig. 1).

The Micromegas (Fig. 2) were produced at CERN using the bulk technology procedure: they have a $128 \mu \mathrm{m}$ gap, $18 \mu \mathrm{m}$ woven stainless steel wire mesh with $63 \mu \mathrm{m}$ pitch, tensioned at about $10 \mathrm{~N}$. A square array of $300 \mu \mathrm{m}$ diameter pillars, with $2 \mathrm{~mm}$ pitch, keeps the micromesh in place, on a PCB specifically designed in Trieste for the COMPASS RICH-1 upgrade.

The anode PCBs are $3.2 \mathrm{~mm}$ thick, organized in 5 layers; they were produced by TVR srl (Vicenza, Italy). After the MM production, they are cut to be $586 \mathrm{~mm}$ long and $283 \mathrm{~mm}$ wide. The square anode pads facing the micromesh have $8 \mathrm{~mm}$ pitch and $0.5 \mathrm{~mm}$ inter-pad clearance and are biased at a positive voltage, supplied via individual $470 \mathrm{M} \Omega$ resistors (one for each of the 4760 anode pads); they are divided in two groups (of 2380 pads), powered by two independent high voltage channels. The micromesh, which is the only non-segmented electrode, is stably kept at ground 
potential, having a significant portion of its surface embedded in conductive glue. Inside the anode PCB, 4760 buried readout pads, facing the anode pads and separated by a $70 \mu \mathrm{m}$ thick FR4 layer from them, transmit the signals with an attenuation of only $\approx 10 \%$, thanks to the large individual capacitive coupling $(40 \mathrm{pF})$ to their corresponding anode pad. This configuration prevents damages to the front-end electronics in case a discharge occurs in the MM, grants a minimal gain drop $(\approx 4 \%)$ in the pads neighboring the discharging one, no discharge propagation and a restoring time of the nominal voltage of $\sim 20 \mu \mathrm{s}$. The MMs are glued in pairs onto the detector holder frame, side by side (Fig. 2) and equipped with readout connectors and bias resistors for the anode pads. The MM gain uniformity distribution, measured using a prototype detector and a ${ }^{55} \mathrm{Fe}$ source, in an $\mathrm{Ar} / \mathrm{CO} 270 / 30$ gas mixture, showed a standard deviation of $\sim 5 \%$.

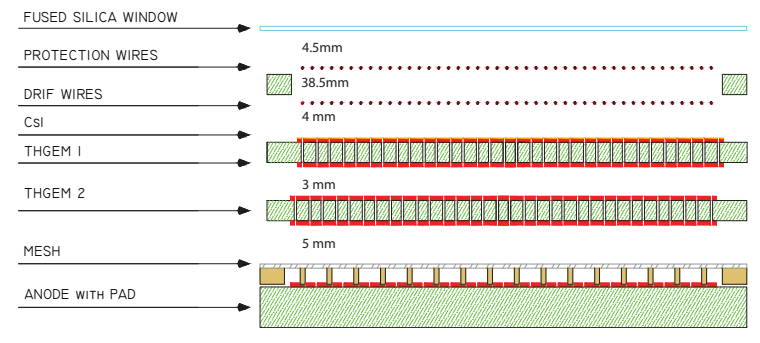

Figure 1: Sketch of the hybrid single photon detector (image not to scale).

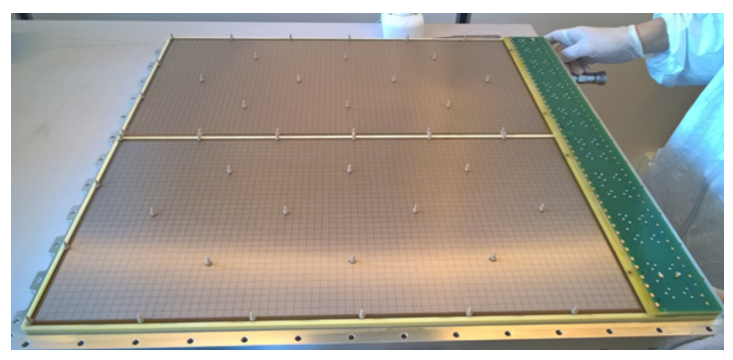

Figure 2: Two Micromegas mounted side by side.

\section{THGEMs production and quality assessment.}

The THGEMs were produced from halogen-free EM 370-5 (Elite Material Co, Ltd.) raw PCB foils. The thickness of the foils (resized to a square of $800 \mathrm{~mm} \times 800 \mathrm{~mm}$ to cut out the less uniform borders) was mapped (in $~ 1300$ points/foil) using a Mitutoyo EURO CA776 coordinate measuring machine: A typical thickness distribution for a good foil presents an average value of $472 \mu \mathrm{m}$ and a standard deviation of $2 \mu \mathrm{m}$. Foils were accepted when $\left(t h_{\max }-t h_{\min }\right) \leq 15 \mu \mathrm{m}$, where $t h_{\max }$ and $t h_{\min }$ are the maximum and minimum of the measured thickness values.

The PCB etching and the drilling of the holes ( 300000 per THGEM) using a multi-spindle machine (Posalux 6000-LZ) were performed by ELTOS S.p.A. (Arezzo, Italy). The quality of the hole edge and walls was granted by replacing the drilling tool every 1000 holes. A specific post production procedure has been applied to all THGEMs [14] to round the hole borders and smooth the surface defects: it consisted in a careful electrode surface polishing, using fine grain pumice powder, high pressure water rinsing and a mild chemical attack by a SONICA PCB solution at $60{ }^{\circ} \mathrm{C}$ in ultrasonic bath, followed by a thermal treatment.

The electric strength and the response of the THGEM under high bias voltage condition were verified in a test setup flushed with an $\mathrm{Ar} / \mathrm{CO}_{2} 70 / 30$ gas mixture where the spark rate was measured: the bias voltage of each sector was automatically increased in steps of $10 \mathrm{~V}$ and the number of discharges (defined as events with current values larger than $50 \mathrm{nA}$ ) was counted. A bias voltage value corresponding to a discharge rate $\leq 1$ event per hour was defined as stable voltage and a THGEM was qualified as electrically stable when all its segments provided a maximum stable voltage exceeding $1200 \mathrm{~V}$ (which corresponds to an effective gain of $\sim 60$ ). The post production 

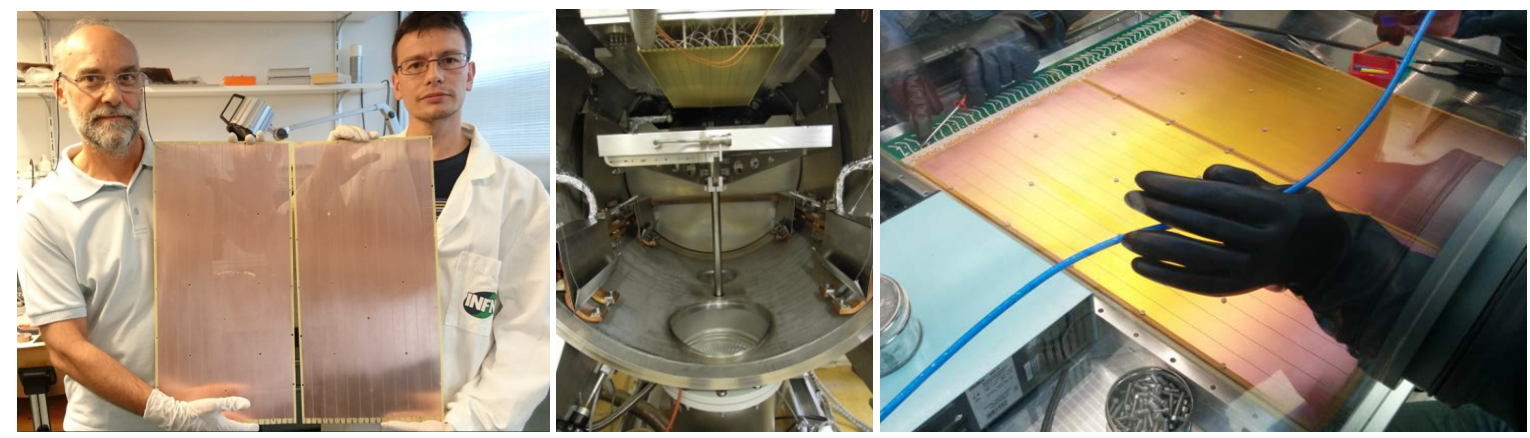

Figure 3: Two THGEMs before the post-production treatment (left), a THGEM inside the CsI evaporation plant (center), THGEMs being mounted on a COMMPASS Hybrid PD inside a glove-box (right).

surface treatment was repeated on THGEMS which failed the first electrical stability validation test. Electrically stable THGEMs were then checked for their gain uniformity imposing a validation threshold $(\sigma \leq 10 \%)$, which was passed by almost all pieces, thanks to the strict thickness uniformity selection criteria previously applied to the raw PCB material.

Qualified THGEMs were then coated with a Ni-Au layer $(\sim 5 \mu \mathrm{m}$ thickness Ni and $0.5 \mu \mathrm{m}$ thickness $\mathrm{Au}$, both chemically deposited at CERN), to preserve the $\mathrm{Cu}$ surface from oxidation and to prepare it as an optimal substrate for the CsI photocathode.

The $300 \mathrm{~nm}$ thick CsI layer was deposited at the CERN Thin Film Laboratory, (Fig. 3, center) following the RD26 procedure [15] (thermal evaporation of CsI at $\sim 1 \mathrm{~nm} / \mathrm{s}$, in a vacuum of $10^{-6}$ mbar $+8 \mathrm{~h}$ at $60{ }^{\circ} \mathrm{C}$ ). A systematic measurement of photocurrent was performed on 60 points for each coated THGEM using VUV light from a $\mathrm{D}_{2}$ lamp to map the response uniformity after the CsI evaporation: good uniformity and small variations between different pieces $(\sigma \sim 10 \%)$ were observed; the overall Q.E. values are compatible with those obtained from the reference sample used for COMPASS and ALICE photocathode production [15], taking into account the THGEM optical transparency of $23 \%$.

\section{Installation and commissioning}

The Hybrid PDs were assembled, equipped and tested before installation; the correct position and planarity of the THGEMs inside the detector is guaranteed by 12 PEEK pillars glued onto corresponding bases of photosensitive material prepared for them on the MM. Auxiliary lateral electrodes, embedded in the chamber frames, are used to correctly shape the electric field at the borders of the chamber volume.

The THGEM photo-cathodes were mounted using a large glove-box built for this purpose. The four MWPC-based PDs to be replaced were part of a unique detector hosting 144 MAPMTs and fused silica lens telescopes too: the MWPCs with CSI photocathodes were dismounted from the RICH-1 vessel and the MAPMT systems were transferred onto the frames of the new hybrid detectors. The combined PDs were installed on COMPASS RICH-1 and equipped with front-end electronics, low voltages, high voltages and cooling services during Spring 2016.

The signals from the readout pads are collected by front-end electronic cards [16] designed for COMPASS RICH-1: they host four APV25-S1 chips, each reading 108 pads. Three front-end cards 
are connected to a 10-bit flash ADC digitizer board equipped with a FPGA performing on-line zero suppression. Data are registered by the COMPASS DAQ [18] and stored for offline analysis. A cooling system using under-pressure water flow assures effcient removal of the heat produced by the readout.

The hybrid PDs operate with an $\mathrm{Ar} / \mathrm{CH}_{4} 50 / 50$ gas mixture; the field configuration used in 2017 is: $0.4 \mathrm{kV} / \mathrm{cm}$ in the Drift region, $1.0 \mathrm{kV} / \mathrm{cm}$ in the two transfer regions between the first and second THGEM and between the second THGEM and the MM. The typical bias applied to the active elements are: $\sim 620 \mathrm{~V}$ to the MM anode, $\sim 1200 \mathrm{~V}$ between top and bottom of THGEMs.

The typical effective gain value is about $2 \times 10^{4}$, larger than the gain provided by the MWPCbased PDs, as can be seen in Fig. 4, left.

A high voltage monitoring program [17] stabilizes the gain by tuning the biases applied to MM and THGEMs to compensate for the environmental changes of temperature and pressure: gain variations, which would be as large as a factor of 2 , are limited to $\sim 5 \%$ over long running periods thanks to this compensation. The IBF to the photocathode has been measured to be $\sim 3 \%$.
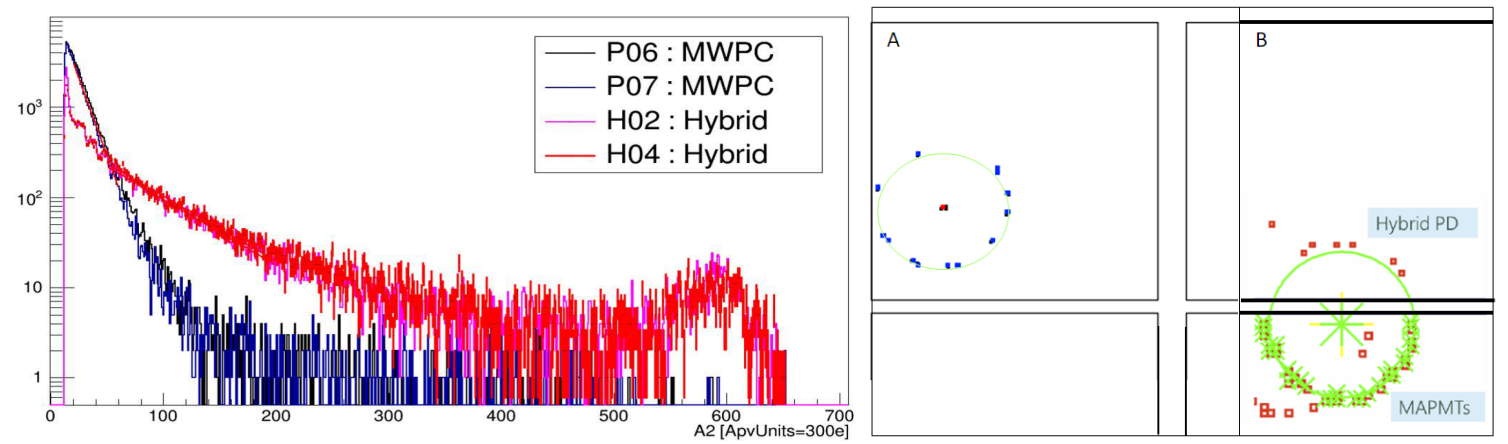

Figure 4: Signal amplitude distributions for MWPCs and Hybrids (left). Two Cherenkov rings from a typcal RICH-1 event in COMPASS 2017 data taking (right).

The COMPASS hybrid PDs have been commissioned in 2016 and provide stable performance in the 2017 run: an example of their Cherenkov rings is presented in Fig. 4, right. The configuration of the capacitive-resistive anode pads allow to operate the MM without inconveniences even in case some anode pads are in short toward ground: this condition was found for two pads during the Hybrid PD tests before installation and for more pads during the commissioning run in 2016 and at the beginning of the 2017 run; this however results in a dead area $<0.1 \%$ of the active area.

Before the 2017 run a refurbishing of the grounding distribution system for the Hybrid PDs was performed: now the typical electronic noise for the new detector channels is $\sim 800$ equivalent electrons r.m.s., stable at a few $\%$ level.

A preliminary evaluation of the number Cherenkov photons detected by the new Hybrid PD's shows an increase with respect to the previously used MWPC-based PDs; the full characterization of the new detectors and the upgraded COMPASS RICH-1 is still ongoing, but indications of a stable and efficient performance of the hybrid PDs are clearly appearing.

The validity of the MPGD-based PD approach for RICH applications is fully confirmed by the successful operation of COMPASS hybrid PDs. 


\section{Acknowledgments}

The activity is partially supported by the H2020 project AIDA2020 GA no. 654168 .

\section{References}

[1] M.Alexeev et al., Nucl. Instr. and Meth. A 639 (2011) 219; S.Dalla Torre et al., Nucl. Instr. and Meth. A 639 (2011) 271; F.Tessarotto et al., JINST 9 (2014) C09011; M.Alexeev et al., Nucl. Instr. and Meth. A 766 (2014) 208.

[2] P. Abbon et al., Nucl. Instr. and Meth. A 577 (2007) 455; P. Abbon et al., Nucl. Instr. and Meth. A 779 (2015) 69.

[3] M.Alexeev et al., Nucl. Instr. and Meth. A 610 (2009) 174; M.Alexeev et al., Nucl. Instr. and Meth. A 617 (2010) 396; M.Alexeev et al., Nucl. Instr. and Meth. A 639 (2011) 130; M.Alexeev et al., Physics Procedia 37 (2012) 781; M.Alexeev et al., JINST 9 (2014) C09017; M.Alexeev et al., JINST 9 (2014) P01006; M.Alexeev et al., JINST 10 (2014) P03026;

[4] M.Alexeev et al., Nucl. Instr. and Meth. A 876 (2017) 96.

[5] W.Anderson et al., Nucl. Instr. and Meth. A 646 (2011) 35.

[6] A.Di Mauro et al., Nucl. Instr. and Meth. A 639 (2011) 274.

[7] L. Periale et al., Nucl. Instr. and Meth. A 478 (2002) 377; P. Jeanneret, PhD thesis, Neuchatel University, 2001; P.S. Barbeau et al., IEEE NS-50 (2003) 1285; R. Chechik et al., Nucl. Instr. and Meth. A 535 (2004) 303; R. Checkik et al., Nucl. Instr. and Meth. A 553 (2005) 35.

[8] A.Breskin et al., Nucl. Instrum. Meth. A 598 (2009) 107; C.D.R.Azevedo et al., JINST 5 (2010) P01002. M.Alexeev et al., Nucl. Instrum. Meth. A 623 (2010) 129. A.Breskin et al., Nucl. Instrum. Meth. A 623 (2010) 132. M.Alexeev et al., JINST 7 (2012) C02014. R.Chechik et al., Nucl. Instrum. Meth. A 595 (2008) 116. G.Hamar et al., Nucl. Instrum. Meth. A 867 (2017) 233.

[9] V.Peskov et al., Nucl. Instrum. Meth. A 695 (2012) 154; R.Alon et al., JINST 3 (2008) P01005; M.Alexeev et al., Nucl. Instr. and Meth. A 732 (2013) 264; M.Alexeev et al., JINST 5 (2010) P03009.

[10] R.Alon et al., JINST 3 (2008) P11001; M.Alexeev et al., Nucl. Instrum. Meth. A 695 (2012) 159.

[11] M.Alexeev et al., JINST 9 (2014) C03046.

[12] M.Alexeev et al., JINST 8 (2013) P01021.

[13] M.Alexeev et al., Nucl. Instrum. Meth. A 824 (2016) 139.

[14] M.Alexeev et al., Nucl. Instr. and Meth. A 766 (2014) 133.

[15] A.Braem et al., Nucl. Instr. and Meth. A 502 (2003) 205; H.Hoedlmoser et al., Nucl. Instr. and Meth. A 566 (2006) 338.

[16] P.Abbon et al., Nucl. Instr. and Meth. A 567 (2006) 104.

[17] J.Agarwala et al. The high voltage system for the novel MPGD-based photon detectors of COMPASS RICH-1, these proceedings.

[18] M. Bodalak et al., JINST 8 (2013) C02009. 